\title{
Synthesis of 2-methoxy-6-(pyrazin-2-ylimino methyl) phenol and its antibacterial activitity
}

\author{
A. A. Jarrahpour ${ }^{1, *}$, M. Motamedifar ${ }^{2}$, N. Hadi $^{2}$, M. Zarei $^{1}$ \\ 1.Department of Chemistry, College of Sciences, Shiraz University, Shiraz 71454, Iran Tel 0098711 \\ 2284822, Fax: 00987112280926 \\ E-mail: aliasghar6683@yahoo.com, jarrah@susc.ac.ir \\ 2.Department of Bacteriology \& Virology, Shiraz Medical School, Shiraz University Medical Science, \\ Shiraz 71345, Iran
}

Received: 30 January 2004 / Accepted: 18 February 2004 / Published: 24 February 2004

Keywords : Schiff base, 2-amino pyrazine, $o$-vanillin, antibacterial activity, heated food.

Pyrazines are found in such heated foods as bread, different meats, baked potatoes and coffee [1], which are formed from serine and thereonine [2]. Pyrazine compounds have shown antifungal [3], antimyco- bacterial [3-4] activities. Furthermore, pyrazine derivatives have been used as antioxidation compounds [3,5-6]. Schiff bases have been used as ligands in complex formation with some metal ions [7]. They have shown biological activities including antibacterial [8-10], antifungal [9-11], anticancer [12-13] and herbicidal [14] activities. On the other hand, Schiff bases have applied as starting materials in the synthesis of a large bioactive and industrial compounds via ring closure, cycloaddition, replacement reactions, etc [15]. In addition, the methoxy group on the molecule enhances the various biological activities [16]. According to above facts, we synthesized 2-methoxy-6-(pyrazin-2-ylimino methyl) phenol $\mathbf{3}$ and then it was tested against four microorganisms Staphylococcus aureus (Gram positive), Bacillus subtilis (Gram positive), Kelebsiella pneumonia (Gram negative) and Pseudomonas aeruginosa (Gram negative). Compound 3 was moderately active against Kelebsiella pneumonia at $200 \mu \mathrm{g}$ and it was inactive against other mentioned bacteria.

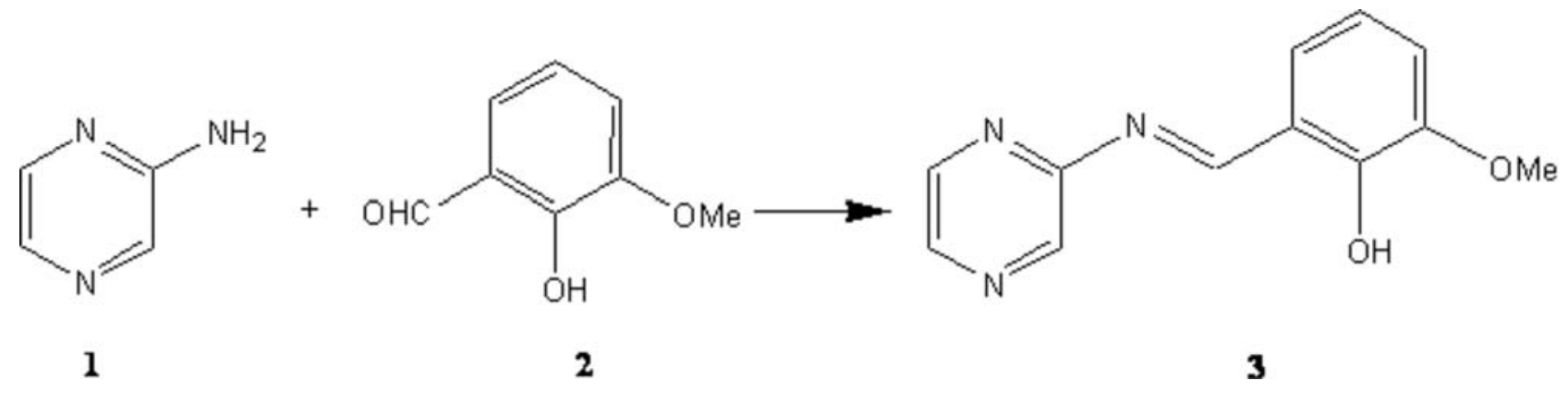

A mixture of 2-amino pyrazine 1 (0.95 g, $10.00 \mathrm{mmol})$, o-vanillin (2-hydroxy-3-methoxy benzaldehyde) 2 $(1.52 \mathrm{~g}, 10.00 \mathrm{mmol})$ and anhydrous sodium sulfate $(3.00 \mathrm{~g})$ in dry dichloromethane $(40.00 \mathrm{~mL})$ was stirred at room temperature for five hours. The suspension was filtered and washed with $\mathrm{CH}_{2} \mathrm{Cl}_{2}$. The solvent was evaporated under reduced pressure and imine $\mathbf{3}$ was formed as a red solid which was recrystalized from methanol (1.90 g, 83\%).

m.p.132-134 ${ }^{\circ} \mathrm{C}$. 
IR $(\mathrm{KBr})\left(\mathrm{cm}^{-1}\right): 1575.73(\mathrm{CH}=\mathrm{N}), 1610.45(\mathrm{C}=\mathrm{N}$ ring), $3190.5-3645.20(\mathrm{OH})$.

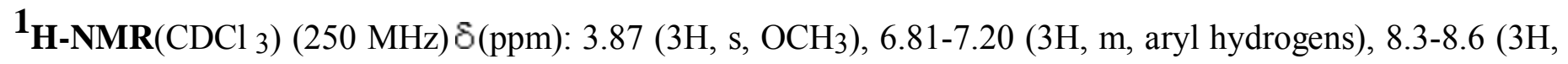
m, pyrazin ring), $9.3(1 \mathrm{H}, \mathrm{s}, \mathrm{N}=\mathrm{CH}), 13.30(1 \mathrm{H}, \mathrm{br}, \mathrm{OH})$.

${ }^{13}$ C- NMR $\left(\mathrm{CDCl}_{3}\right)(62.90 \mathrm{MHz}) \delta(\mathrm{ppm}): 56.64\left(\mathrm{OCH}_{3}\right), 116.47-142.90$ (aryl and pyrazine carbons), 167.30 $(\mathrm{N}=\mathrm{CH})$.

MS (m/z, \%): $229\left(\mathrm{M}^{+}, 60.3\right), 150\left(\mathrm{HOPhOCH}_{3} \mathrm{CH}=\mathrm{N}, 22.0\right), 135\left(\mathrm{HOPhOCH}_{3} \mathrm{C}, 18.0\right), 106\left(\mathrm{C}_{4} \mathrm{~N}_{2} \mathrm{H}_{3} \mathrm{~N}=\mathrm{C}_{\text {, }}\right.$ 14.0), $79.0\left(\mathrm{C}_{4} \mathrm{~N}_{2} \mathrm{H}_{3}, 44.4\right), 43\left(\mathrm{C}-\mathrm{OCH}_{3}, 100.0\right), 41(\mathrm{~N}=\mathrm{CH}-\mathrm{N}, 96.0)$.

\section{Acknowledgment}

The authors thank the Shiraz University Research Council for financial support (Grant No. 81-SC-1540-C220 ).

\section{References}

1. Buchbauer, G.; Klein, C.Th.; Wailzer, B.; Wolschann, P. J. Agric. Food Chem. 2000, 48, 4273-4278.

2. Chi-Kuen Shu J. Agric. Food Chem. 1999, 47, 4332-4335.

3. Dolezal, M.; Miletin, M.; Kunes, J.; Kralova, K. Molecules 2002, 7, 363-373.

4. Gezginci, M. H.; Martin, A. R.; Franzblau, S. G. J. Med. Chem 2001, 44, 1560-1563.

5. Jeanjot, P.; Bruyneel, F.; Arrault, A.; Gharbi, S.; Cavalier, J.-F.; Abels, A.; Marchand, C.; Touillaux, R.; Rees, J.-F.; Marchand-Brynaert, J. Synthesis 2003, (4), 513-522.

6. Cavalier, J.-F.; Burton, M.; De Tollenaere, C.; Dussart, F.; Marchand, C.; Rees, J.-F.; MarchandBrynaert, J. Synthesis 2001, (5), 768-772.

7. Tai, X.; Yin, X.; Chen, Q. and Tan, M. Molecules 2003, 8, 439-443.

8. Kabeer, A . S.; Baseer, M. A.; Mote, N. A. Asian J. Chem. 2001, 13 (2), 496-500.

9. More, P. G.; Bhalvankar, R . B.; Pattar, S. C. J. Indian Chem. Soc. 2001, 78 (9), 474-475.

10. Pandeya, S. N.; Sriram, D.; Nath, G.; De Clercq, E. IL Farmaco 1999, 54, 624-628.

11. Singh, W. M.; Dash, B. C. Pesticides 1988, 22 (11), 33-37.

12. Hodnett, E. M. and Dunn, W. J. J. Med. Chem. 1970, 13, 768-770.

13. Pathak, P.; Jolly, V. S.; Sharma,K. P. Orient. J. Chem. 2000, 16(1), 161-162.

14. Samadhiya, S.; Halve, A. Orient. J. Chem. 2001, 17 (1), 119-122.

15. Aydogan, F.; Öcal, N.; Turgut, Z. and Yolacan, C. Bull. Korean Chem. Soc. 2001, 22, 476-480.

16. Halve, A.; Goyal, A. Orient. J. Chem. 1996, 12(1), 87-88.

Sample Availability : Available from MDPI.

(C) 2004 MDPI. All rights reserved 